intracranial aneurysms is overall more prevalent following the results of several randomized trials, but surgery continues to play an important role in management of some subsets of aneurysms. Here, we describe the clinical outcomes of coiling vs clipping for ruptured intracranial aneurysms utilizing a national database.

Methods We utilized the TriNetX Analytics Network to query 2,740 cases up to March $27^{\text {th }}, 2021$. We identified patients $>18$ years old who underwent coiling or clipping for the treatment of ruptured intracranial aneurysm using ICD-10 codes of I60.1, I60.2, I60.3, I60.6, and I60.7. Patient demographics, baseline characteristics, comorbidities, and clinical outcomes were evaluated within 1-year postoperatively. Mortality and adverse outcome analyses were performed after propensity score matching to control for baseline characteristics and comorbidities between the two cohorts. A combined pooled and individual analyses of the above ICDs were conducted to investigate the differences in clinical outcomes due to the aneurysm's location.

Results The coiling cohort consisted of 2011 patients with mean age $56.8 \pm 13.9$ and $30.9 \%$ male and the clipping

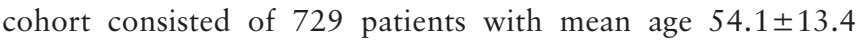
and $28.4 \%$ male. After propensity matching for baseline characteristics and comorbidities, a total of 666 patients were included in each cohort. The incidence of surgical/ medical complications, ICU, and vasospasm, were significantly lower in the coiling cohort ( $\mathrm{p}$-value $=0.008,0.03$, 0.03 ) compared to the clipping cohort within 1-year postoperatively using the combined ICD codes. There was no significant difference in 1-year-mortality between the two cohorts. Additionally, individual ICD code analysis revealed that coiling of anterior communicating artery (ACoA) aneurysm was associated with significantly less surgical/medical complications and hydrocephalus ( $p$-value $=0.0008,0.015)$, and coiling of posterior communicating artery (PCoA) aneurysms was associated with significantly less vasospasm treatment ( $p$-value $=0.034)$ as compared to the respective clipping cohort. There was no significant difference in 1year-mortality between the two cohorts by location of the aneurysm using individual ICDs.

Conclusion This national database analysis of 1,332 propensity-matched patients undergoing coiling or clipping after a ruptured intracranial aneurysm provides evidence that 1-year clinical outcomes, including surgical/medical complication, and vasospasm, favored coiling. In particular, ACoA-coiled patients had significantly less hydrocephalus, and PCoA-coiled patients had significantly lower vasospasm treatment within 1-year as compared to clipping.

Disclosures A. Nia: None. R. Lall: None. P. Kan: None. V. Srinivasan: None.

\section{0-009 SHORT-TERM OUTCOMES FOR ELECTIVELY TREATED UNRUPTURED INTRACRANIAL ANEURYSMS IN HEALTHY ADULTS: A FEASIBILITY STUDY FOR AMBULATORY NEUROINTERVENTIONAL PROCEDURES}

M McPheeters*, M Waqas, H Rai, K Rho, A Gong, E Levy. Neurosurgery, University at Buffalo, Buffalo, NY

10.1136/neurintsurg-2021-SNIS.9

Background Unruptured intracranial aneurysms are a relatively common disease entity with a relatively uncommon, yet potentially devastating, risk of rupture. Over the past three decades, the safety of neurointerventional techniques for the treatment of intracranial aneurysms has greatly improved and allow for rapid post-operative recovery. Despite this, neurointerventional treatments remain almost exclusively inpatient procedures. The purpose of this study is to assess the short-term safety profile of electively treated intracranial aneurysms in healthy patients as part of a feasibility study for the ambulatory treatment of intracranial aneurysms.

Methods A retrospective analysis was performed on 200 sequential healthy adult patients with intracranial aneurysms treated via neurointerventional techniques in an elective fashion at a tertiary care center. Healthy adults were classified as having a CCI $\leq 4$ and $\mathrm{mRS} \leq 1$. Patients with non-saccular aneurysm (e.g. fusiform, dissecting), posterior circulation aneurysms, and/or multiple aneurysms treated in the same setting were excluded from analysis. A descriptive analysis was then performed on outcome measures, including, intraoperative complications, 48-hour post-op clinical event, length of stay, and 30-day mRS.

Results A total of 176 patients were included in the analysis with an average age of 52 years and an average CCI of 1.6. Aneurysm location included 116 ICA aneurysms (65.9\%), 24 ACA aneurysms (13.6\%), 21 MCA aneurysms (11.9\%), and 15 PCoA aneurysms (8.5\%). Neurointerventional techniques included primary coil $(\mathrm{n}=37,21.0 \%)$, stent-coil $(\mathrm{n}=38$, $21.6 \%)$, intravascular flow diversion $(n=96,54.5 \%)$, and intrasaccular flow diversion $(n=5,2.8 \%) .4$ patients $(2.3 \%)$ experienced intraoperative complications. 14 patients $(8.0 \%)$ experienced a clinical event in the 48-hour post-operative period, with $9(5.1 \%)$ of these being clinically significant. The most common clinically significant event was a groin hematoma $(n=3)$. The highest proportion of clinically significant events occurred in the stent-coil group $(4 / 38 ; 10.5 \%)$. The average length of hospital stay was 1.7 days. At 30-day follow-up only 1 patient $(0.6 \%)$ had a documented mRS higher than prior to intervention.

Conclusions The neurointerventional treatment of unruptured intracranial aneurysms in healthy patients is associated with a low risk of peri-operative and short-term complication. These results suggest that with properly selected patients, the neurointerventional treatment of intracranial aneurysms may be feasible in the ambulatory setting and further prospective analysis is recommended.

Disclosures M. McPheeters: None. M. Waqas: None. H. Rai: None. K. Rho: None. A. Gong: None. E. Levy: None.

\section{0-010 RACIAL DISPARITY IN MECHANICAL THROMBECTOMY UTILIZATION: MULTICENTER REGISTRY RESULTS FROM 2016-2020}

${ }^{1} \mathrm{~A}$ Wallace*, ${ }^{1} \mathrm{D}$ Gibson, ${ }^{2} \mathrm{~K}$ Asif, ${ }^{3} \mathrm{D}$ Sahlein, ${ }^{4} \mathrm{~S}$ Warach, ${ }^{5} \mathrm{~T}$ Malisch, ${ }^{6} \mathrm{M}$ Lamonte. ${ }^{1}$ Neurointerventional Surgery, Ascension Columbia St. Mary's Hospital, Milwaukee, WI; ${ }^{2}$ Neurointerventional Surgery, Ascension Columbia St. Mary's Hospital, Arlington Heights, ILi ${ }^{3}$ Neurological Surgery, Goodman Campbell Brain and Spine, Carmel, IN; ${ }^{4}$ Neurology, University of Texas, Austin, TX; ${ }^{5}$ Interventional Neuroradiology, AMITA Health Medical Group, Arlington Heights, IL; ${ }^{6}$ Neurology, University of Maryland, Baltimore, MD

\subsection{6/neurintsurg-2021-SNIS.10}

Background and Purpose Previous studies on racial disparity in mechanical thrombectomy (MT) treatment of acute large vessel occlusion (LVO) stroke lacked individual patient data 